\title{
Respiratory Function Monitoring During Mechanical Ventilation in Pediatric Intensive Care Unit
}

\author{
Praveen Khilnani
}

\begin{abstract}
Noninvasive monitoring of lung function during mechanical ventilation has been used to study disease processes causing respiratory failure. Pediatric pulmonary function monitoring during mechanical ventilation in the pediatric intensive care unit in patient with respiratory failure is becoming more common in western countries. The article describes a review of principles and methods of respiratory function monitoring in the pediatric age group. It attempts to incorporate experience from original articles previously published as well as the experience in monitoring pulmonary mechanics at our institution. Routine monitoring of pulmonary mechanics in patients being mechanically ventilated may have significant impact on morbidity and/or mortality of pediatric patients with respiratory failure.

Key articles pertaining to lung function testing in pediatric age group from past 15 years were used as well as clinical experience encountered by attending pediatric intensivists at Henrico Doctors' Hospital was incorporated.

Over the past ten years the use of monitoring of gas exchange and bedside pulmonary mechanics monitoring as a part of respiratory function monitoring has become more common. With better clinical information to precisely document the status of lung function, it may lead to improved methods of ventilator management which may prevent complications and may significantly impact on morbidity and/or mortality of mechanically ventilated pediatric patients. (Indian J Pediatr 1998; 65 : 409-418)
\end{abstract}

Key words: Monitoring; Pulmonary function; Pediatric intensive care.

The use of noninvasive methods for the determination of mechanical properties of the respiratory system in artificially ventilated patients has been advocated for the study of disease processes causing respiratory failure.

Knowing the mechanical properties of the lung and the dynamics of lung-ventilator interaction should allow the optimum matching of mechanical support to the mechanical need.

Rosen et $a l^{1}$ showed the effects of bedside pulmonary mechanics testing during

Reprint requests : Praveen Khilnani, M.D. FAAP, Columbia/HCA Healthcare Corporation, Henrico Doctors' Hospital 1602 Skipwith Rd., Richmond, Virginia 23229, USA. infant mechanical ventilation in a retrospective analysis. In 251 neonates ventilated with the help of pulmonary mechanics testing, there was a significantly lower incidence of pneumothorax and intraventricular hemorrhage when compared to 217 neonates who were managed using similar ventilators without the help of pulmonary mechanics testing.

Fisher et al have used pressure volume loops to identify and relieve lung distention during infant mechanical ventilation. Although, currently, there are no studies available that directly show the effect of pulmonary function testing of ventilated patients on outcome in the pediatric ICU based on neonatal experience the applica- 
tion of pulmonary mechanics should take pediatric ventilation from "Good Judgement" to an "Informed Judgement" style of management which may have significant impact on morbidity and/or mortality of mechanically ventilated pediatric patients.

In our pediatric ICU at Henrico Doctors' Hospital, monitoring pulmonary mechanics is a routine part of monitoring respiratory functions using a Bicore CP-100 (Irvine, California) Pulmonary Function Monitor on all intubated pediatric patients with respiratory failure in addition to monitoring of pulse oximetry, end tidal $\mathrm{CO}_{2}$, arterial blood gases and $\mathrm{FiO}_{2}$.

Monitoring of respiratory function essentially includes :

(a) monitoring gas exchange.

(b) monitoring respiratory mechanics

It is important for a clinician to remember that regular clinical examination and use of radiographs to follow the progress of lung disease cannot be replaced by pulmonary function testing in ventilated patients. Equally important is the fact that ICUs frequently have a real problem of data overload with advancing technology. The goal of respiratory monitoring should not be to produce overwhelming data which are not useful and unnecessarily divert the clinician's attention from more important issues. Instead, the goal should be to analyze and process date in such a way so to improve clinical decision-making.

Preferably, monitoring should be continuous, painless, as non-invasive as possible, and should require minimal or no patient cooperation. Collection, display and recording of data should be automated. Accumulated data should be useful with regard to patient management.

Monitoring of Gas Exchange : For patients in respiratory failure, gas exchange can be monitored by frequent monitoring of arterial blood gases with continuous monitoring of $\mathrm{FiO}_{2}$, pulse oximetry and end tidal $\mathrm{CO}_{2}\left(\mathrm{EtCO}_{2}\right)$, if available. In this discussion, we will concentrate on monitoring of respiratory mechanics, i.e., pulmonary function testing during mechanical ventilation.

Monitoring of Respiratory Mechanics : Ideal techniques of monitoring respiratory mechanics should be simple, accurate, noninvasive and should provide continued free access to the patient. The parameters measured with respiratory mechanics monitoring during mechanical ventilation include:
(A) Airway pressure (Paw)
1. Peak airway pressure
2. Inadvertent PEEP
3. Mean airway pressure
(B) Flow (V)
(C) Tidal volume $\left(\mathrm{V}_{\mathrm{T}}\right)$
(D) Esophageal pressure (Pes)
(E) Pressure volume curves
(F) Flow volume loop
(G) Compliance - Dynamic compliance (Cdyn), Respiratory system compli- ance (Crs)
(H) Resistance (R)
(I) Time constraint (TC)
(J) Work of breathing (WOB)

Airway pressure (Paw) : Peak airway pressure is pressure required to deliver a given tidal volume at a given rate and is affected by flow resistive and elastic properties of lung and chest wall.

1. Transpulmonary pressure $=$ Paw - Pes (pleural pressure). Paw sampled at inlet of endotracheal tube represents proximal airway pressure.

2. Inadvertent PEEP : Positive end ex- 
piratory pressure generated in the alveoli with air trapping during spontaneous or mechanical ventilation.

Flow (V) is defined as volume of gas flowing across a given point per unit time. Flow is affected by air passage resistance, viscosity, humidity, temperature and mix of gas.

Tidal Volume $\left(\mathrm{V}_{\mathrm{T}}\right)$, inspiratory and expiratory breath volume, is a function of gas flow and respiratory rate.

Esophageal pressure (Pes) is measured by balloon catheter placed in mid-esophagus. Pes is used as a reasonable approximation of pleural pressure $(\mathrm{Ppl})$ in older children. In neonates with a distended chest wall Pes may not correlate with Ppl.

Pressure volume curve : Airway pressure is plotted against tidal volume (Fig. 1).

Flow volume loop : Inspiratory and expiratory flow is plotted against volume (Fig. 2).

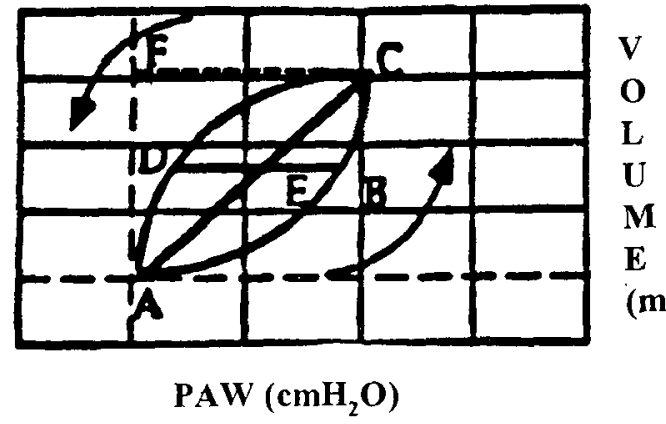

o

$\mathbf{L}$ $\mathbf{M}$ (mL)

Fig. 1. Pressure-volume loop under ideal conditions during one respiration cycle. $A B C$ reflects the inspiratory changes in volume and pressure and $C D A$ represents expiratory. Points EB and $D E$ represent the midpoints of each phase and can be used to calculate pulmonary resistance by dividing their value by flow. Work of breathing is calculated by measuring the area of $A B C$ FA. (Adapted from Bancalari E. Pulmonary function testing... in Neonatal Pulmonary Care. Thibeault, DW. Gregory GA. eds. 1979 Addison-Wesley, Reading, MA).

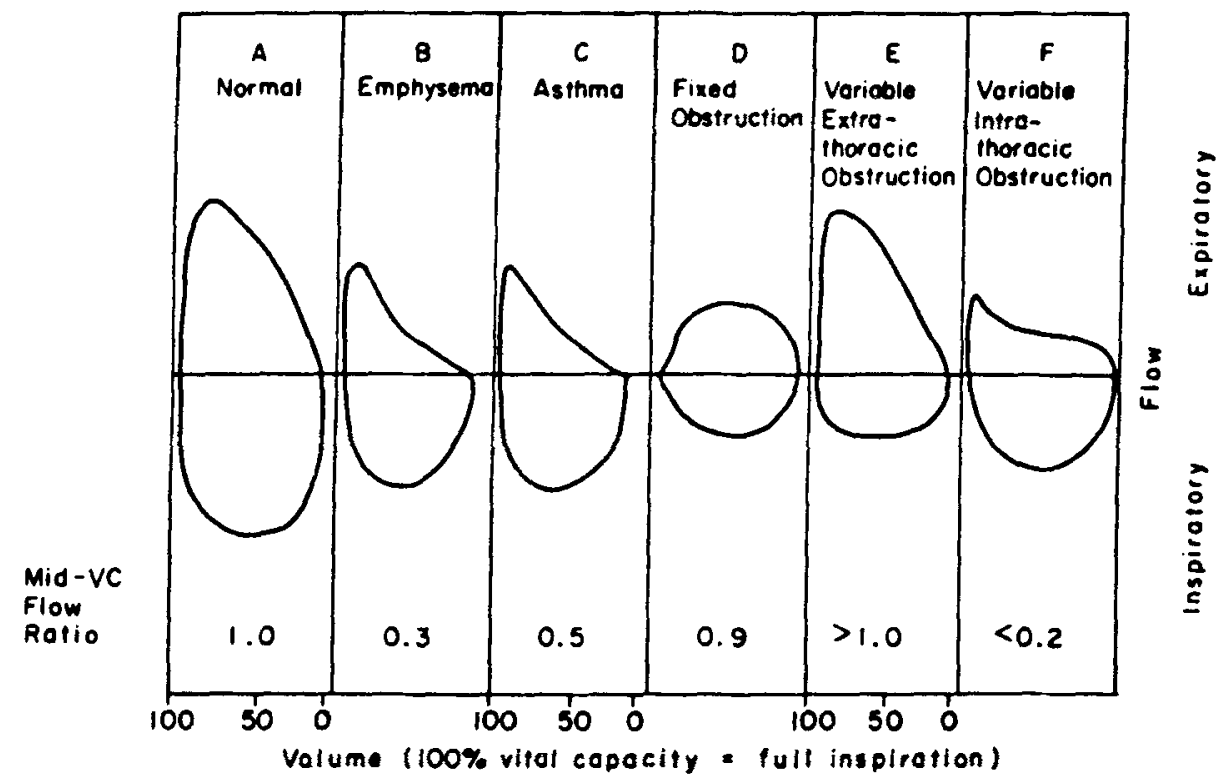

Fig. 2. Flow-volume loops in various conditions (modified from Miller and Hyatt). VC = vital capacity 
Compliance $(\mathrm{C})$ is the measure of stiffness or distensibility $(\mathrm{L} / \mathrm{cm})$. Dynamic compliance (Cdyn) is tidal volume divided by total change in pressure required to deliver that volume. It is dependent on elastic (elastance and volume) as well as flow resistive (resistance and flow) properties of

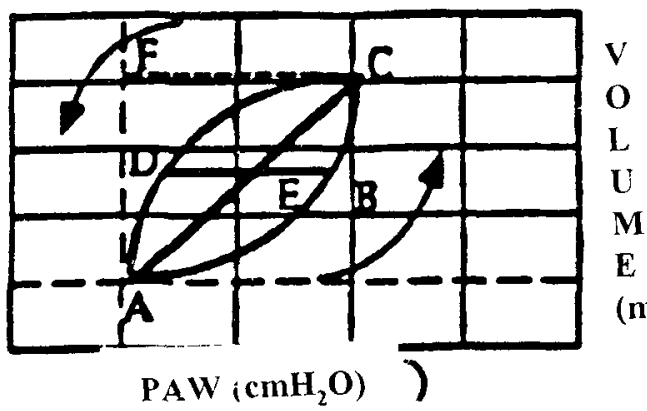

Fig. 3. Pressure volume loop work of breathing is calculated by measuring the area of ABCFA.

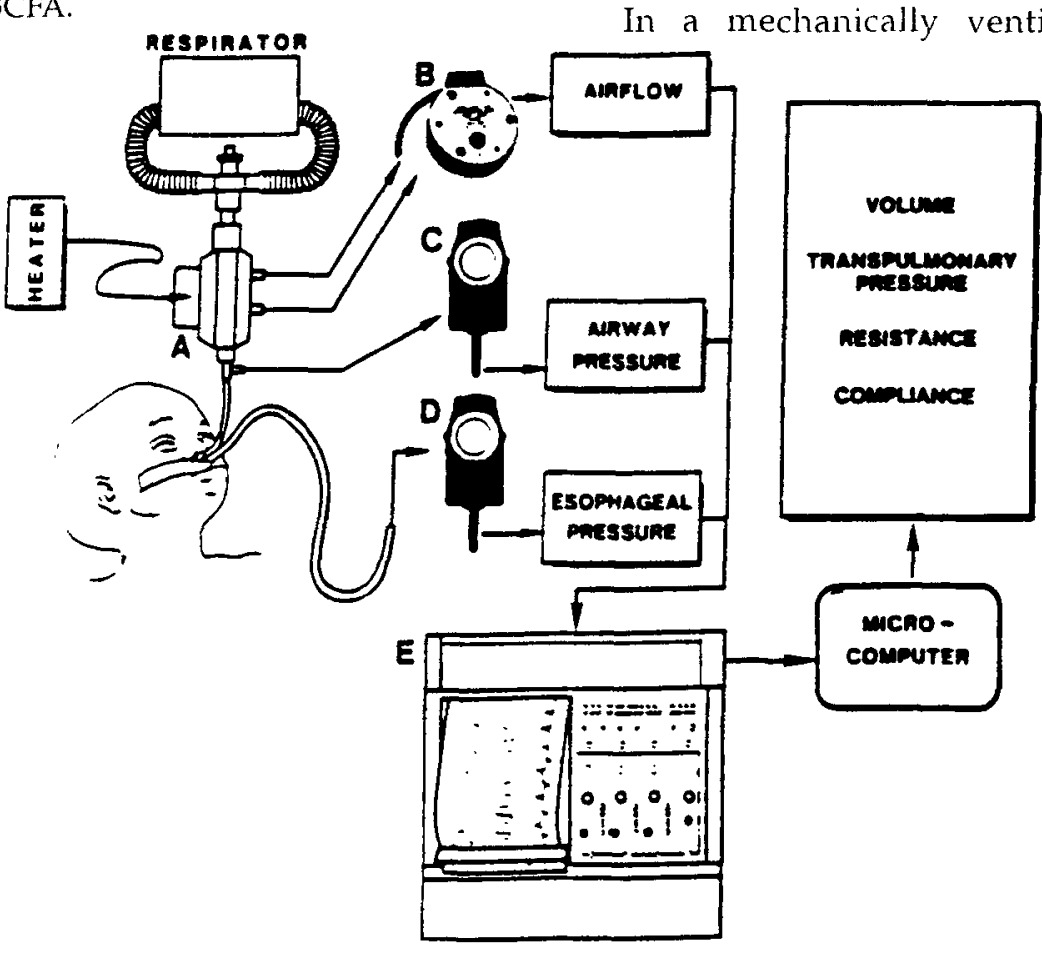

the lungs.

Resistance ( $R$ ) is pressure divided by flow $(\mathrm{cm} / \mathrm{L} / \mathrm{sec})$.

Time constant (TC) is compliance $X$ resistance (sec).

Time required to fill/empty an alveolus One time constant fills it $63 \%$

Two time constants fill it $87 \%$

Three time constants fill it to $95 \%$

Work of breathing (WOB) (Fig. 3).

Area ABCFA as shown in this pressure volume curve is equal to the work of breathing. Increased resistance increases the work of breathing.

Technique of Respiratory Mechanics Monitoring (Fig. 4)

In a mechanically ventilated child,

Fig. 4. Lonfiguration of bedside pulmonary tunction testing devices: A. Pneumotachograph; B. Differential transducer for airflow; $C$. and D. Transducers for airway and esophageal pressures. 


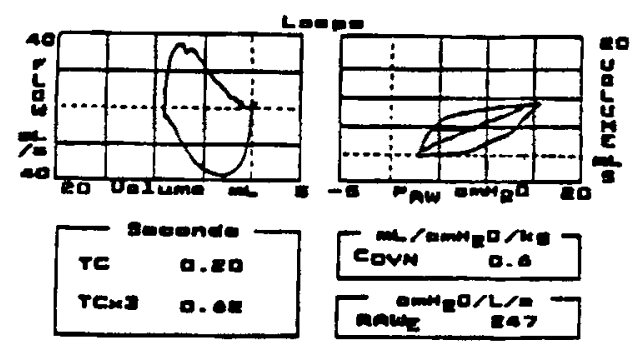

Fig. 5. Shows flow volume loop recording by Bicore CP-100 on left and pressure volume loop recording on right. Time constant (TC), compliance (Cdyn) and airway resistance $\mathrm{RAW}_{\mathrm{E}}$ numbers are also displayed.

Example: The Bicore CP-100 Pulmonary Function Monitor comes with a Varflex flow transducer; smart valve occlusion valve and a smart cath esophagus balloon catheter.

flow/ pressure transducer connected at the endotracheal tube connection with the ventilator circuit is an essential component of respiratory mechanics monitoring. Airway pressure (Paw), as well as Flow (V), can be accurately measured using this transducer.
For measurements of compliance, resistance and time constant, an occlusion valve may be added to the ventilator circuit enabling pressure and volume measurements in addition to flow measurement. An esophageal balloon catheter placed in midesophagus connected to the pulmonary function monitor measures Pes, which, in the absence of chest wall distortion, is considered in close approximation with pleural pressure (Ppl). Data from the transducer and esophageal balloon are processed by computerisation of the pulmonary function monitor and resulting pulmonary function parameters, e.g., compliance, resistance, mean airway pressure and time constants can be printed. Flow volume loops may also be obtained. The recorded display produces tracings of changes in airway pressures, esophageal pressure, airflow and tidal volume for each breath. Computer assisted breath-to-breath studies can also be accomplished (Figs. 5, 6, 7)

The flow transducer in line near the endotracheal tube measures two key variables :

Airway pressure (Paw) and Flow (V)

The addition of the occlusion valve to the

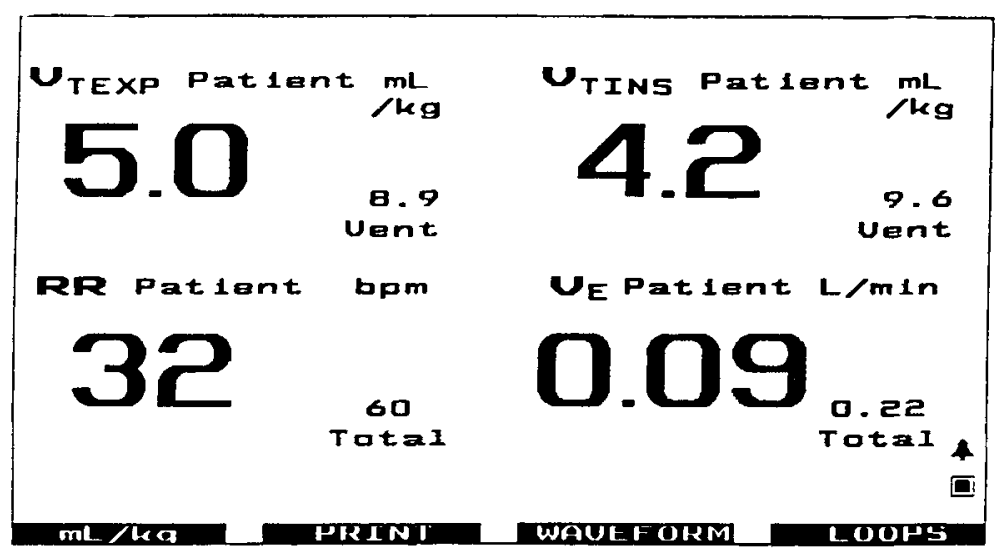

Fig. 6. The monitor allows evalution of both $\mathrm{Vt}$ as well as minute volume on a continuous basis. 


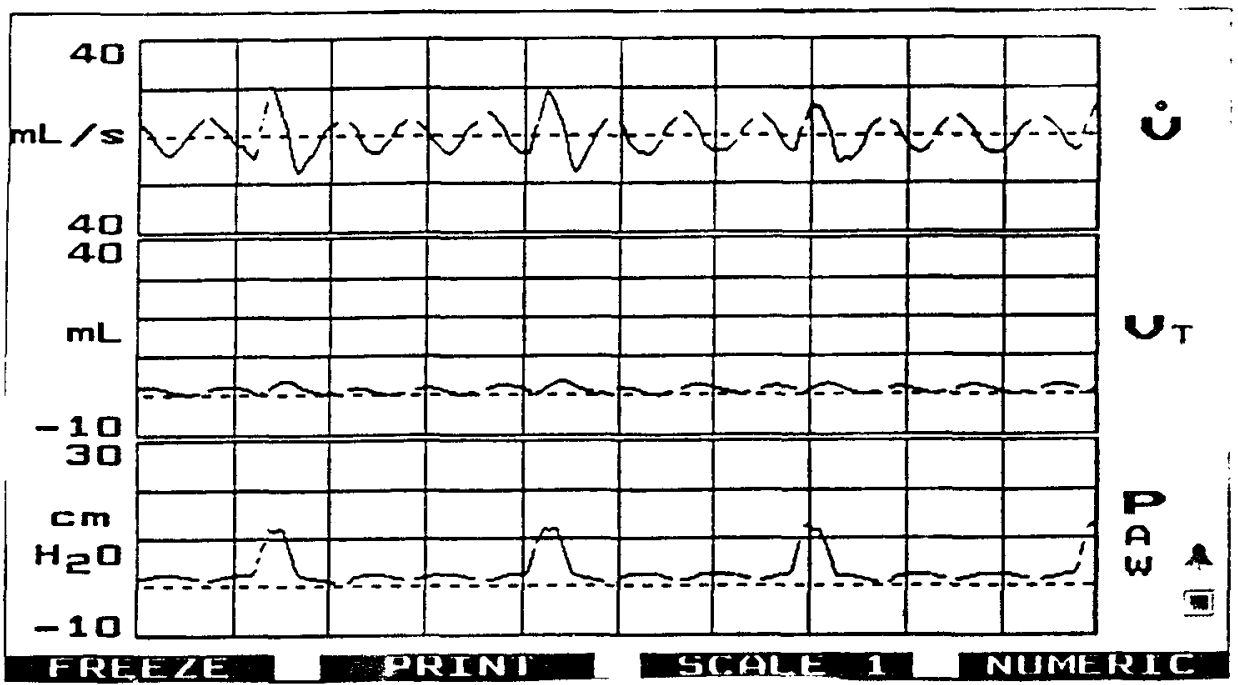

Fig. 7. CP-100 neonatal display scaler graphs of airway pressure, volume and flow. It demonstrates a patient/ventilator synchrony.

circuit enables measurement of : Airway pressure (Paw); Compliance (CRS); Respiratory resistance (RRS); Flow (V); Total resistance (RTS); Expiratory time constant.

The addition of the esophageal balloon catheter with tip placed in mid-esophagus enables measurement of pleural pressure in children and adults. (There is controversy regarding its accuracy in neonates due to distortion of chest wall.) , $^{3,4.5}$

Calculated parameters : RR (Respiratory rate); PIP (Peak inspiratory pressure); PEEP (Positive end expiratory pressure); VE (Minute ventilation); Mean airway pressure, Peak inspiratory flow rate and expiratory flow rate; Inspired and expired tidal volume; Inspiratory and expiratory time.

\section{Clinical Applications and_Significance}

Clinical applications and significance of respiratory mechanics monitoring in mechanically ventilated patient can be illus- trated by the following examples:

(A) Flow Volume Loop is a plot of inspiratory flow and inspiratory flow against volume on same paper.

1. Fixed upper airway obstruction (fig. 2) : tracheal stenosis, undersized endotracheal tube, kinked or plugged endotracheal tube can be detected because both expiratory and inspiratory parts of the flow volume loop look flat, i.e., reduced flow during inspiration as well as expiration.

2. Variable extrathoracic obstruction (fig. 2) : flat peak flow during inspiration.

3. Variable intrathoracic obstruction (fig 2) : flat peak flow during expiration.

4. Restrictive lung disease (fig. 2) : decreased flow and tidal volume during inspiration.

5. Asthma (fig. 2) : decreased peak 

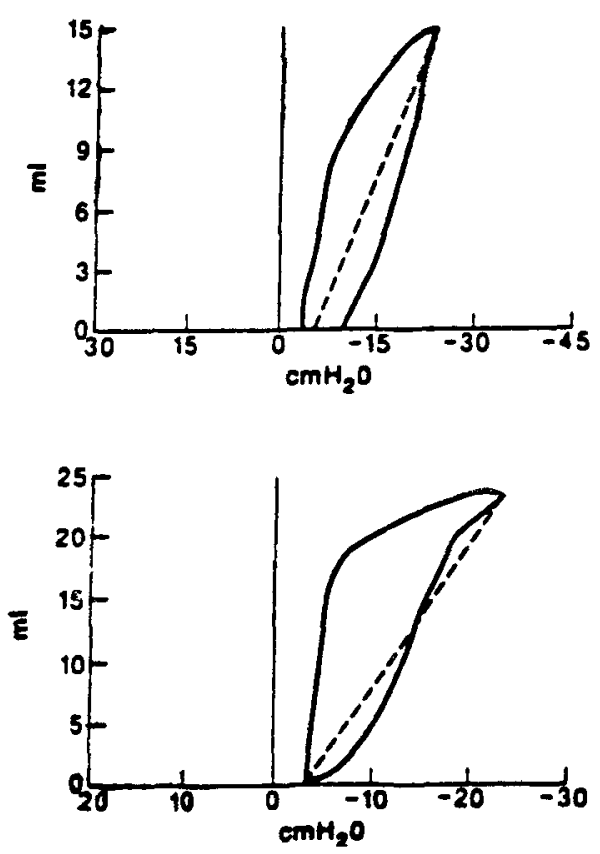

Fig. 8. Volume-pressure loops reflecting normal distention (top) and overdistention (bottom).

flow in maximal expiratory flow volume curve.

(B) Pressure volume curve is airway pressure plotted against tidal volume.

1. Normal (Fig. 1)

$A C$ represents inspiration.

$C A$ represents expiration.

2. Airway resistance $=$ Paw at $D$ divided by flow at $D$.

3. Area $A B C F A$ represents work of breathing.
(a) Lung overdistention (Auto PEEP).
(b) Decreased compliance : pres- sure curve is bent to side.
(c) Increased expiratory resistance : inspiratory pressure volume curve is concave.

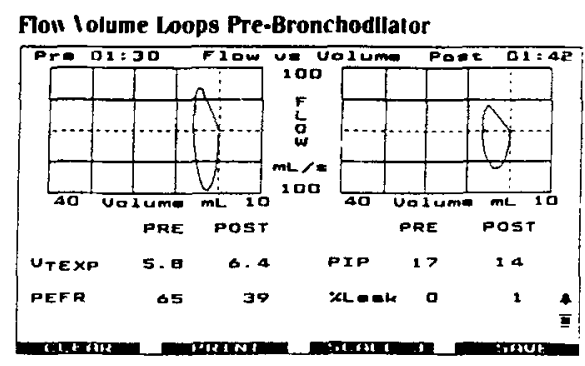

Fig. 9. Flow volume loop showing comparison of tracing pre- and post-bronchodilator treatment. PEFR = Peak expiratory flow rate, $\mathrm{PIP}=$ Peak inspiratory pressure, VT EXP $=$ Expired tidal volume.

Pulmonary function monitor generates flow volume and pressure volume loops and with the help of these, changes in work of breathing, compliance, resistance as well as airway obstruction and over distension of lung can be detected (fig 8).

(C) Compliance can be calculated by obtaining single occlusion passive flow volume curve. Extrapolation of flow volume loop to zero flow is done. Taking volume at zero flow divided by occlusion pressure gives compliance. Bicore CP-100 calculates C20 (i.e., compliance for the terminal $20 \%$ of inspiration). A ratio of $\mathrm{C} 20$ to $\mathrm{C}>1$ is normal. However, if the ratio is $<0.8$, this may indicate overdistension.

(D) Resistance as measured by the Varflex Flow Transducer in Bicore CP 100 monitor increases in asthma, partial obstruction or a kink in endotracheal tube, and bronchopulmonary dyspiasia. Normal values in children are 20 $40 \mathrm{~cm} \mathrm{H}_{2} \mathrm{O} / \mathrm{L} / \mathrm{sec}$. A change in airway resistance may help determine effectiveness of bronchodilator therapy (fig. 9). Increased airway resistance increases work of breathing in an intubated patient who has spontaneous breath- 


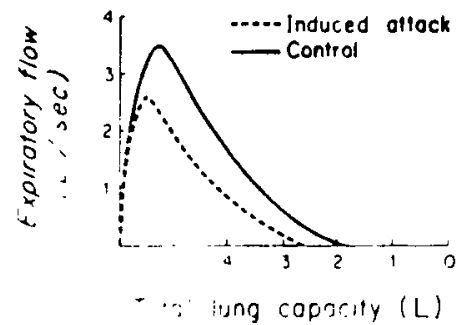

Fig. 10. Maximal expiratory flow-volume curves from a subject with asthma. The patient was asymptomatic during the control period. Symptoms including wheezing were induced by inhaling an extract of ragweed. The resulting change in lung mechanics is readily identified from maximal expiratory flow volume curves.

ing in addition to being mechanically ventilated. Auto PEEP occurs when expiratory resistance increases, e.g., asthma (figs. 10, 11).

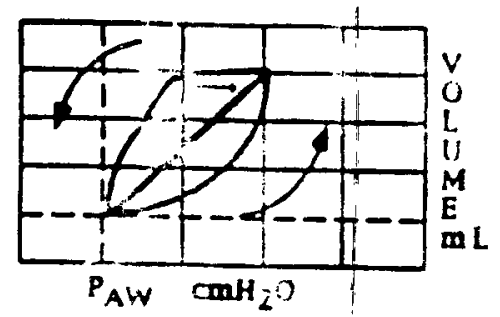

Fig. 11. Expiratory resistance-increased resistance during the expiratory phase is identified by distinct bowing with regard to the "ideal" compliance line.

(E) Time constant (fig. 12) is the time taken to fill an alveolar unit to $63 \%$ of its capacity. It is calculated by multiplying compliance and resistance. If measured compliance is $0.004 \mathrm{~L} / \mathrm{cm}$ and resistance is $30 \mathrm{~cm} \mathrm{H}_{2} \mathrm{O} / \mathrm{L} / \mathrm{sec}$ then time constant (TC) is $0.12 \mathrm{sec}$.
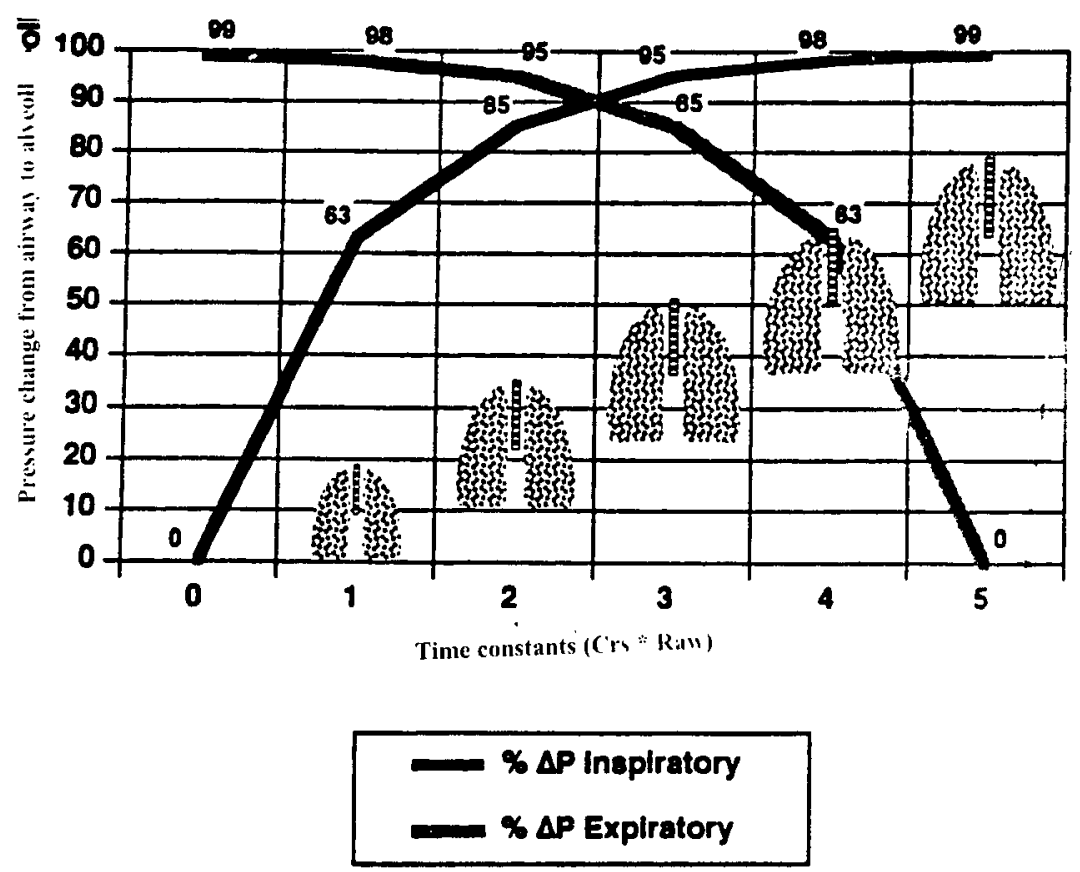

Fig. 12. 


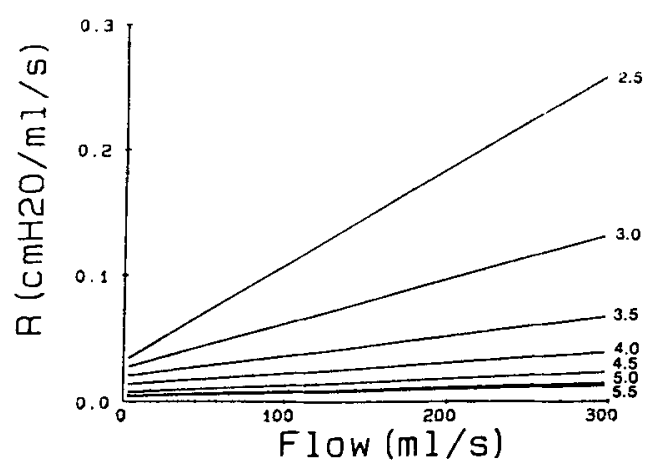

Fig. 13. Resistance of pediatric endotracheal tubes (Sheridan uncuffed, Argyle, NY) plotted as a function of flow in the expiratory direction. Tubes are uncut, and tube size (internal diameter in millimetes) is indicated on the curves.

Since 5 time constants are required to fill the alveolar unit to $98 \%$ of its capacity, inspiratory time selected on the ventilator should be at least $0.6 \mathrm{sec}-$ onds.

Caution : It has been shown that airway pressures displayed on ventilator have poor correlation with actual delivered airway pressure.

Effect of Endotracheal Tube (Fig. 13)

Resistance posed by an endotracheal tube is flow dependent. This may inadvertently decrease the peak flows. The flow volume loop may be distorted in the presence of an endotracheal tube; a correction needs to be made before resistance and compliance is calculated. Pulmonary function testing has not been found useful for predicting successful extubation in neonatal RDS. ${ }^{6}$ In another study, compliance and oxygen transport had no relationship. Respiratory system compliance and
$\mathrm{PaO}_{2}$ did not predict PEEP level for maximal oxygen transport. ${ }^{7 s}$ Uses of Bedside Pulmonary Mechanics in
Mechanically Ventilated Patient

A. Detect lung overdistension/prevent pneumothorax.

B. Identify patient ventilator dysynchrony.

C. Track rapid changes in tidal volume and compliance.

D. Detect endotracheal tube obstruction, right mainstem intubation, bronchospasm and pneumothorax.

E. Assess efficacy of bronchodilator therapy.

F. Monitor tidal volume, minute ventilation for spontaneous as well as mechanical breaths.

G. Other potential uses :

1. effectiveness of pressure support ventilation.

2. assessment work of breathing.

3. measure muscle strength by measuring maximal inspiration and expiratory pressures.

\section{CONCLUSIONS}

1. Routine pulmonary function testing on mechanically ventilated pediatric patients is still in infancy and done only at major tertiary pediatric intensive care units.

2. Neonatal pulmonary function studies have demonstrated benefit in prevention of pneumothorax and intraventricular hemorrhage in critically ill neonates.

3. More pediatric studies are needed to show long-term effect on changes in morbidity and patient outcomes. It is 
reasonable to apply neonatal experience to try and take pediatric ventilation from "Good Judgement" to an "Informed Judgement" style of management which may have significant impact on morbidity and/or mortality of mechanically ventilated pediatric patients.

\section{REFERENCES}

1. Rosen CW, Mammel MC, Fisher JB, et al. The effects of bedside pulmonary mechanics testing during infant mechanical ventilation : A retrospective analysis. Pediatr Pulmonol 1993; 16 : 147-152.

2. Fisher JB, Mammel MC, Coleman JM, et al. Identifying lung overdistension during mechanical ventilation using volume pressure loop. Pediatr Pulmonol 1988; 5 : 10.14.

3. LeSouef PN, Lopes JM, England SJ, et al. Influence of chest wall distortion on esophageal pressure. J Appl Physiol 1983; $55: 353-358$.

4. Heaf DP, Turner H, Stocks J, et al. The accuracy of esophageal pressure measurements in convalescent and sick intubated infants. Pediatr Pulmonol 1986; $2: 5-8$.

5. Asher MI, Coates AL, Collinge JM, et al. Measurement of esophageal pressure in neonates. J Appl Physiol 1982; 52 : 491-494.

6. Veness-Meehan KA, Richter S, Davis JM. Pulmonary function testing prior to extubation in infants with respiratory distress syndrome. Pediatric Pulmonol 1990; $9: 2-6$

7. Sivan Y, Deakers TW, Newth CJH. Effect of positive and expiratory pressure on respiratory compliance in children with acute respiratory failure. Pediatr Pulmonol 1991; $11: 103-107$.

8. Witte MK, Galli SA, Chatburn RL, et al. Optimal positive end expiratory/pressure therapy in infants and children with acute respiratory failure. Ped Res 1988; $24: 217-221$.

\section{FURTHER READING}

1. England SJ. Current techniques for assessing pulmonary function in the newborn and infant. Pediatr Pulmonol 1988; 4 : 48-53.

2. Pfaff JK, Morgan WJ. Pulmonary function in infants and children. Ped Clin North Am, 41 : 401-423.

3. Sly PD, Brown KA, Bates JH. Noninvasive determination of respiratory mechanics during mechanical ventilation of neonates. A review of current and future techniques. Pediatr Pulmonol 1988; 4 : 39 47.

4. Hyatt RE, Black LF. The flow volume curve a current perspective. Am Rev Resp Dis 1973; 107 : 191-199.

5. Boysen GP, Broome JA. Noninvasive monitoring of lung function during mechanical ventilation. Crit Care Clin $1988 ; 4: 5-27-541$. 